response function (Fig. 1). Also, inspection of the graph suggested that "probability tracking" occurred as predicted from the generalized statistical learning theory (Estes, 1957, 1959).

The "lag" in probability tracking, a theoretical constant $v$ which was expected to emerge after initial trials, is represented in Fig. 2.

The initial estimate of $v$ from the data was 10.5 trials; however, Estes (1957) implied that this was a biased estimate, since it included lag decrement following the periodic slope reversals of the $\pi_{n}$ function and the subsequent crossover of that function with the $P_{n}$ function. Thus, the apparent periodic decrements in tracking lag around Trials 30,130 , and 230 are actually adjustments for functional slope reversals. Considering this factor, the obtained value of tracking "lag" in Fig. 2 was relatively constant over trials, as expected.

Since $v$ is an inverse function of the stimulus sampling ratio $\Theta$, an estimate of that parameter would yield a more efficient estimate of v. Estes (1957) stated that $\Theta$ can be estimated from the slope of a terminal block of trials of an obtained response function, within a period.

Using Eq. 2, mean estimates of the stimulus sampling ratio $\Theta \cong .074$, and of its inverse, mean tracking lag $v \cong 13.5$ trials, were obtained. These estimates, although not unbiased, were considered adequate for most experimental purposes.

$$
\text { Eq. 2. } \quad \bar{P}_{j}(k, m) \approx a_{j}-\frac{b_{i}}{\theta}+\frac{b_{j}}{2}(2 m k-k+1)
$$

$k=$ number of trials within a block. $\mathrm{m}=$ block within a period.

For this case, $k=50$ trials and $m=2$ (i.e., the second block of 50 trials within each 100-trial period) (from Estes, 1957).

The achievement of the relatively strong probability tracking effect with a stable tracking lag was apparently highly consistent with the predictions of the generalized (Estes, 1957, 1959) statistical learning theory of choice behavior.

\section{REFERENCES}

EDWARDS, W. Probability learning in 1000 trials. Journal of Experimental Psychology, 1961,62, 385-394.

ESTES, W. K. Theory of learning with constant, variable, or contingent probabilities of reinforcement. Psychometrika, 1957, 22, 113-132.

ESTES, W. K. The statistical approach to learning theory. In S. Koch (Ed.), Psychology: A study of a science. Vol. 2. New York: McGraw-Hill 1959. Pp. 380-491.

ESTES, W. K., \& STRAUGHAN, J. H. Analysis of a verbal conditioning situation in terms of statistical learning theory. Journal of Experimental Psychology, 1954, 47, 225-234.

HUMPHREYS, L. G. Acquisition and extinction of verbal expectations in a situation analogou to conditioning. Joumal of Experimental Psychology, 1939, 25, 294-301.

\section{NOTES}

1. A preliminary report of this study was read by the author at the Spartan Psychological Association Meeting, May 20, 1968, San Jose, California

2. Now at the University of Oregon, Eugene Oregon 97403. This study was conducted under the supervision of Professor Robert S. Witte.

\title{
Trait similarity and trait evaluation as correlates of attraction
}

\begin{abstract}
ABRAHAM TESSER, University of Georgia, Athens, Ga. 30601

The finding that trait similarity and attraction are correlated may be difficult to interpret. Research has indicated that attraction to another is related to the evaluative aspect of the beliefs about Other (i.e., to the extent that Other is believed to have positive attributes, he will be better liked) and that persons tend to attribute positive traits to themselves. To the extent that persons do this, both the similarity and evaluation hypotheses make the same prediction. In assessing the relative importance of each, both were found to make significant contributions to the prediction of attraction with similarity being less important than evaluation.
\end{abstract}

In the social psychological literature, some 0 bearing on those same attributes, one would predict that the more similar $\mathrm{O}$ is to $P$ the more attractive $P$ should find $O$.If, however, in fact, the predicted relationship is found there is some question about its meaning.

Cronbach (1958) has convincingly argued against the uncritical use of dyadic (e.g., similarity) hypotheses. The “... principal difficulty is that interpretations dealing with interactions [e.g., similarity data] can be advanced meaningfully only after the simpler main effects associated with the perceiver or the object of perception have been given separate consideration [p. 355]."

"Consider the hypothesis relating ... [similarity to attraction]. Perhaps ... [attraction] can be predicted directly from the self-perception of . . [P] . . considered alone, perhaps from the perceived characteristics of ... [O] . . . considered alone, or from a linear combination of the two. An interaction hypothesis, i.e., a second-degree relationship, is justified only if it improves significantly on these simpler predictions [p. 356]."

One could plausibly argue that firstdegree relationships can explain the link between trait-similarity and attraction. Let us focus for the moment on P's cognitions about $O$ 's traits. There is a good deal of data indicating that one can predict P's attraction to $O$ just on the basis of the way he evaluates the traits he believes to be charcteristic of $O$. That is, one can sum P's evaluative responses to each of $O$ 's traits or average them in some way and predict his attraction to $\mathrm{O}$ (e.g., Fishbein, 1965).

If it is also true that $P$ tends to attribute positively evaluated traits to himself (Edwards, 1957) then the relationship between $\mathrm{P}-\mathrm{O}$ similarity and P's at traction to $\mathrm{O}$ may be spurious. Take, for example, the limiting case when $\mathbf{P}$ indicates that all the positively evaluated attributes on some list are characteristic of himself and all the negatively evaluated attributes are not. Then, to the extent that $O$ is cognized to have positively evaluated attributes and not to have negatively evaluated attributes he will be more similar to $P$. In this case, both the first-order hypothesis (i.e., evaluation of $O$ 's traits) and the second-order hypothesis (i.e., P-O similarity) would predict more attraction to $O$. If the second-order hypothesis doesn't add any predictive efficiency, Cronbach (1958) would argue that we accept the more parsimonious first-order hypothesis. It is possible and plausible, however, that both sources of variance operate simultaneously and predictive efficiency is maximized when using both.

If the argument is that $P$ bases his attraction toward $O$ on an evaluation of $O$ 's attributes rather than P-O similarity, a viable 
strategy would be to examine the zero-order correlations $r_{s-a}$ (i.e., the correlation between similarity and attraction) and $\mathrm{r}_{\mathrm{o}-\mathrm{a}}$ (i.e., the correlation between P's evaluation of $O$ 's traits and attraction) and the correlations partialling out $P$ 's evaluation of O's attributes $\left[r_{s-a(o)}\right]$ or P's similarity to $O\left[r_{a-a(s)}\right]$. If $P$ is attending to both similarity and evaluation of $O$ 's attributes, the expectation is:

(1) $r_{s-a}>0$ and $r_{o-a}>0$

(2) $r_{s-a(o)}>0$ and $r_{o-a(s)}>0$

If $\mathrm{P}$ is attending only to similarity:

(1a) $\mathbf{r}_{\mathrm{s}-\mathbf{a}}>0$ and $\mathbf{r}_{\mathbf{0}-\mathbf{a}} \geqslant 0$

(2a) $r_{s-a(o)}>0$ and $r_{0}-a(s)=0$

If $\mathrm{P}$ is attending only to his evaluation of $\mathrm{O}$ 's attributes:

(lb) $\mathrm{r}_{\mathrm{s}-\mathrm{a}} \geqslant 0$ and $\mathrm{r}_{\mathrm{o}-\mathrm{a}}>0$

(2b) $r_{s-a(o)}=0$ and $r_{0-a(s)}>0$

If, in fact, $P$ is attending to both similarity and an evaluation of $O$ 's attributes one could use a linear regression model with attraction as the criterion and compare the relative contribution of each in a linear prediction scheme. One might also include P's evaluation of his own attributes as Cronbach suggests.

\section{METHOD}

Fifty-four male undergraduates at a large midwestern university volunteered to serve as Ss.

Each $\mathrm{S}$ was individually exposed to four descriptions of "Others." These descriptions were supposedly constructed by clinical psychologists from a battery of tests $\mathrm{O}$ had taken. Each description indicated how probable it was that $O$ possessed each of 12 traits. ${ }^{2}$ The descriptions were actually constructed by varying the probabilities of each trait such that two descriptions would be likeable and two would be dislikeable. ${ }^{3}$ (A more complete discussion of stimulus construction and experimental procedure can be found in Tesser, 1967.)

When a $S$ arrived, he rated the likeability of the 12 traits on two five-point semantic differential scales (Likeable-Dislikeable; Nice-Awful). He was then given a description to examine for up to $2 \mathrm{~min}$. The description was taken away and the $S$ indicated his beliefs (Fishbein, 1965) about $\mathrm{O}$ by rating each trait on two five-point belief scales (Likely-Unlikely; ImprobableProbable). The $\mathrm{S}$ was told that this was not a memory test but rather a way of recording his impression. After recording his beliefs, $S$ 's attraction to $O$ was measured on two five-point semantic differential scales (Likeable-Dislikeable; Nice-Awful). This procedure was repeated three more times with the remaining three descriptions. Description order was randomized for each $S$.

Each $S$ then made some more ratings of the traits, looked at the descriptions a second time, recorded his beliefs about each a second time, and made some more global ratings of each. Since all of these second ratings are irrelevant to the present hypothesis, they will not be discussed further except to note that half the Ss made these ratings before the attraction ratings and half after the attraction ratings.

Finally, all Ss indicated their beliefs about themselves in terms of the 12 traits on the two belief scales (Likely-Unlikely; Improbable-Probable).

An index of evaluation of O's traits, similarity of $S$ and $O$, and $S$ 's self-evaluation had to be constructed. While there are any number of ways of constructing each of these indices, the ones chosen are as follows.

The index of similarity was a Fisher's $z$ transformation of the product-moment correlation of beliefs about self with belief about $\mathrm{O}^{4}$ across the 12 traits. The index of evaluation of $O$ 's traits was a weighted sum of the evaluation of traits where the weights are the beliefs 4 (probability) that 0 possesses each trait (Fishbein, 1965). A similar index was constructed for the evaluation of S's traits, i.e., the sum of the evaluation of traits weighted by belief that self possesses each trait.

\section{RESULTS AND DISCUSSION}

Correlations between the similarity index and attraction to $O\left(r_{s-a}=.38 ; p<.002\right)$ and evaluation of $O$ 's traits and attraction $\left(r_{o-a}=.57 ; \mathrm{p}<.002\right)$ were computed over the 54 Ss over the four descriptions. Thus the $\mathrm{N}$ for each correlation is 216.5 The partial correlations were also computed, i.e., $I_{S-a(0)}=.17[t(213)=2.556 ; p<.04]$ and $I_{0-a(s)}=.48 \quad[t(213)=7.902 ; p<.002]$ All of these correlations are significantly different from zero and follow the hypothesized pattern of Ss attending to both similarity and evaluation of $O$.

An analysis of regression was used to determine the relative weights of the two predictors and to see if evaluation of one's own traits adds significantly to the prediction of attraction. The results of this analysis confirm the story told by the zero and first-order correlations above. The best single predictor of attraction to $O$ is an evaluation of $O$ 's traits. Similarity, while statistically significantly $[\mathrm{F}(1,213)=6.593$; $.10>p>05]$ boosting the multiple $R$ (from .566 to .583) accounts for very little additional predictable variance. The addition of evaluation of own traits did not improve prediction $[F(1,212)<1]$.

What these data suggest is that a rather simple, first-order relationship can account for a larger portion of the attraction variance than the second-degree relationship. That is, the way $P$ evaluates $O$ 's traits is more highly related to attraction than is similarity of P's and $O$ 's traits.

This is not to say that the hypothesized relationship between trait similarity and attraction is completely untenable. First of all, even when evaluation of $O$ 's traits were statistically held constant, similarity is significantly related to attraction. From a me thodological point of view the indicant of similarity may have been poor. Basically, the measure of similarity was the correlation of beliefs about self with beliefs about $O$. This measure is only sensitive to differences in profile shape and not height or dispersion. Ss may be sensitive to the latter two and thus the similarity attraction relationship would be attenuated. Even if evaluation of O's traits is more important a determinant of attraction than similarity, it is possible that similarity is a determinant of evaluation. That is, all things being equal, traits that are similar to own traits may be more positively evaluated than dissimilar traits.

It should also be pointed out that the indicant of P's evaluation of $O$ 's traits may also be such that its relationship with attraction is attenuated. The indicant assumes Ss simply add the evaluative components of each trait. Other information processing strategies are possible. For example, $S s$ may average the components, weigh them differentially, or use a multiple cut-off kind of strategy to mention a few.

\section{REFERENCES}

CRONBACH, L. J. Proposals leading to analytic treatment of social perception scores. In $R$. Tagiuri and L. Petrullo (Eds.), Person perception and interpersonal behavior. Stanford: Stanford University Press, 1958.

EDWARDS, A. L. The social desirability variable in personality assessment and research. New York: Holt, Rineh art, \& Winston, 1957.

FISHBEIN, M. A consideration of beliefs, attitudes, and their relationships. In I. D. Steiner and M. Fishbein (Eds.), Current studies in social psychology. New York: Holt, Rinehart, \& Winston, 1965.

SECORD, P. F., \& BACKMAN, C. W. Social psychology. New York: MoGraw-Hill, 1964. TESSER, A. Attitude and relevance for action mediated by belief complexity as determinants of action. Unpublished doctoral thesis, Purdue University, 1967.

1. Sometimes attraction is found to vary with trait complementerity rather than similarity. This paper, however, is concerned with interpreting the latter relationship when it turns up.

2. The traits were unconventional, materialistic emotional, skeptical, meticulous, guilty, impul sive, domineering, distrustful, idealistic, demanding, and optimistic.

3 . There were actually three sets of four (two likeable, two dislikeable) descriptions varying in complexity. However, in the interests of brevity only the analysis across complexity conditions will be discussed.

4. While the present report is based on beliefs about $O$, a phenomenological index, the results are essentially the same when they are based on what actually appeared in the description.

5 . The $\mathrm{N}$ used for all analy ses was 216 . However, since the observations were based on only $54 \mathrm{Ss}$, each of the 216 observations are not independent. To partially compensate for this nonindependence all of the $p$ values reported in this paper have been doubled. 